\title{
„ES IST IRRITIEREND, UNTER ETWAS ZU LEIDEN, DAS KEINEN SCHMERZ VERURSACHT". ZU STEPHAN THOMES LITERARISCHEN AUSLOTUNGEN DES PREKÄREN BEGEHRENS
}

\begin{abstract}
It is annoying to suffer from something that causes one no pain" - in regard to Stephen Thomes' literary attempts of insight into precarious desire

The essay is devoted to the novels of the German writer Stephen Thome. The writer's narrative attention is focused on the puzzlement regarding intimate relations between males and females, aiming at capturing to what extent love, "a warming and intoxicating drug in the social coldness" (Volkmar Sigusch) is capable of making life meaningful. In the very centre of the analysis stands on the one hand the "in-each other-reflection' of the male and female positions, on the other hand the palpable ambivalence between the inner freezing of the figures and their desire for gentle intimacy. The attempt has been made in order to open the arranged forms of the fulfillment of the desire - i.e. love and sexuality - to the possibility of depicting the phenomenon which is - according to Sigusch - significant for the late-modern era: more important than the sexual act for the partners is a steady relationship in which they feel accepted and preserved.
\end{abstract}

KEYWORDS: Stephan Thome, love, sexuality, desire, students' revolt

„Es ist irritierend, unter etwas zu leiden, das keinen Schmerz verursacht. Nur ein winziges Ziehen, ein sanfter Druck, der sich kaum lokalisieren lässt und den jede Aktivität umgehend zum Verschwinden bringt. Aber in den Pausen ist er da. Immer"1 - mit diesen Worten bringt der Protagonist in Stephan Thomes Debütroman Grenzgang (2009) sein Grundgefühl auf den Punkt, das in einer ähnlichen Form auch in den darauffolgenden Romanen Fliehkräfte (2012) und Gegenspiel (2015) thematisiert wird. Die narrative Aufmerksamkeit gilt den Aporien in den FrauMann-Beziehungen, um einzufangen, inwiefern Liebe, eine „erwärmende Rausch-

\footnotetext{
${ }^{1}$ Stephan Thome: Grenzgang. Roman. Frankfurt a. M. 2009, S. 160f. In der Folge als G mit einfacher Seitenzahl zitiert.
} 
droge in der gesellschaftlichen Kälte“(Volkmar Sigusch) ${ }^{2}$, dem Leben einen Sinn zu geben vermag. Thomes Figuren scheinen kaum etwas von der Wärme zu spüren, vielmehr klagen sie über „,das plötzliche Einfrieren von inneren Regungen“ (G 403), das nicht zuletzt darauf zurückgeht, dass es ihnen schwer fällt, von der Freiheit Gebrauch zu machen. „Sie müssen“, so Lothar Struck in der Rezension von Grenzgang, „den Augenblick des Gefühls ,ergreifen“ und ihr Begehren in die Dauer der Liebe verwandeln. Sie müssen - wählen". ${ }^{3}$

Das Interesse richtet sich im Folgenden zunächst auf die Figurationen des Begehrens in Thomes vielbeachtetem Erstling Grenzgang, im zweiten Schritt wird vergleichend auf andere Werke eingegangen. Einen zentralen Aspekt der Analyse bildet einerseits die vom Autor geschilderte Ineinanderspiegelung weiblicher und männlicher Positionen, andererseits die im Gesamtwerk greifbare Ambivalenz zwischen dem innerem Einfrieren und einem ,stürmische[n] Verlangen nach einer Umarmung, das [...] eine Form stummer Gegenwehr [ist], vielleicht den hektischen Bewegungen ähnlich, mit denen Ertrinkende schließlich beschleunigen, wovor sie sich zu retten versuchen“ (G 409). Der folgende Versuch zielt darauf ab, die inszenierten Realisierungsformen des Begehrens - Liebe und Sexualität - ansatzweise auf die Darstellung des Phänomens hin zu öffnen, das Volkmar Sigusch als den für die Spätmoderne signifikanten „Weg von der Wollust zur Wohllust“ bezeichnet: „Wichtiger als der sexuelle Akt ist eine feste Beziehung, in der die Partner sich angenommen und aufgehoben fühlen“. ${ }^{4}$

\section{1.}

Gegenwärtig ist unser Alltag von sexuellen Reizen ebenso gesättigt wie entleert. Das ist eine der zentralen Paradoxien der so genannten sexuellen Revolution der letzten fünfzig Jahre. Offenbar wird sexuelle Lust durch deren übertriebene kulturelle Inszenierung, durch deren beinahe lückenlose Kommerzialisierung und elektronische Zerstreuung wirksamer ausgetrieben, als es die alte Unterdrückung durch Verbote vermocht hat. ${ }^{5}$

Im Fazit der Studie Neosexualitäten. Über den kulturellen Wandel von Liebe und Perversion weist Sigusch im Hinblick auf Sexualität auf eine paradoxale Verschiebung hin, die an einer anderen Stelle Marie-Luise Angerer beschreibt, wenn sie „,von einem Begehren, das sich aus dem Mangel speist, zu einem Begehren, das der Über-

\footnotetext{
${ }^{2}$ Volkmar Sigusch: Neosexualitäten. Über den kulturellen Wandel von Liebe und Perversion. Frankfurt a. M./New York 2005, S. 18.

${ }^{3}$ Lothar Struck: Provinzkritiker. Aufschlußreiche Bemerkungen zu einer Pseudokritik über Stephan Thomes Buch „Grenzgang “, die den Zustand der bundesdeutschen Literaturkritik beleuchten [online], <http://www.glanzundelend.de/Artikel/artikelalt/provinzkritiker.htm> [29.11.2015].

${ }^{4}$ Sigusch: Neosexualitäten, S. 23.

${ }^{5}$ Ebd., S. 170f.
} 
fülle des Daseins geschuldet ist" ${ }^{\text {‘6 }}$, ausgeht. Um die Folgen davon darzustellen, beruft sich Angerer zum einen auf Slavoj Žižek, der von anorektischem Verhalten eines Subjekts spricht, das sich selbst den grenzenlosen Genuss verbietet. Zum anderen greift sie auf Theoreme von Paul Verhaeghe zurück, der ein solches Paradox als grundlegend für eine postindustrielle Gesellschaft beschreibt: Wenn dem Einzelnen alles gestattet ist und er zugleich unter Zwang steht, dies ausnutzen zu müssen, breiten sich statt Glück nur Langeweile oder Depressionen aus. Die große Freiheit sowie das schrankenlose Genießen führen, schlussfolgert Angerer, in eine Leere, was annehmen lasse, Einschränkungen seien offenbar nötig, um jene Kraft am Leben zu erhalten, die die Psychoanalyse als Sexual- und Todestrieb bezeichnet habe, und die seit Jaques Lacan als Begehren das Subjekt vor sich her treibe. ${ }^{7}$

Man bemerkt, dass unabhängig von der jeweiligen Optik Sigusch wie Angerer einstimmig auf neue Zwänge inmitten neuer Freiheiten verweisen und an Verboten/Ritualen/Grenzen eine Antriebskraft für das Begehren festmachen: „Der Reiz kommt aus dem Verbot" ${ }^{8}$ In diesem Licht betrachtet, trifft Stephan Thome in Grenzgang den Nerv der Zeit. Zwar antizipiert der Titel das Überschreiten (ergo: Vorhandensein) von Grenzen, in die Geschichte wird aber in wesentlichen Zügen die signifikante Paradoxiesemantik eingebaut: Von den Protagonisten heißt es, sie laufen ,im Kreis, weil gerade an Grenzgang niemand wusste, wo die Grenze eigentlich verlief" (G 227).

Im Mittelpunkt des Plots steht eine sich anbahnende Liebesbeziehung zwischen zwei Mittvierzigern - einem alleinstehenden Gymnasiallehrer Thomas Weidmann, der Angst hat ,vor der schrittweisen Transformation in eine geile Kröte, das Objekt von Schülerspott und besorgt getuschelten Bemerkungen von Kollegen“" (G 233), sowie einer geschiedenen Hausfrau Kerstin Werner, zu deren Gefühlschaos ihr in einem neuen Familienglück aufblühender Ex-Mann, der pubertierende Sohn und die demente Mutter gleichermaßen beitragen. Über Jahre hinweg kreuzen sich die Wege der Figuren, bevor sie zaghaft zueinander finden. Die erzählten Ereignisse aus dem Zeitraum, in dem sich die beiden näher kommen, wechseln mit Reminiszenzen an ihre früheren Liebeserfahrungen.

Eine Achse, um die herum die Story organisiert ist, bildet das titelgebende Volksfest. Sein Prinzip besteht darin, alle sieben Jahre die Ortsgrenzen gemeinschaftlich abzulaufen. Den Erzählrhythmus bestimmen die Leitworte (Der Stein..., ...die Grenze..., ...in Ewigkeit) aus Festansprachen, welche metaphorisch eine Diagnose über moderne Befindlichkeiten vorwegnehmen, freilich in Form eines Wunschbildes. Je lauter der Grenzgang von Festrednern als „eine Tradition [bejubelt wird], in der sich die Verbundenheit ausdrückt zwischen gestern, heute und morgen,

\footnotetext{
${ }^{6}$ Marie-Luise Angerer: Vom Begehren nach dem Affekt. Zürich/Berlin 2007, S. 8.

${ }^{7}$ Ebd., S. $96 f$.

${ }^{8}$ Sigusch: Neosexualitäten, S. 101.
} 
zwischen den Generationen“, als „,die Feier all dessen, [...], was uns die Gewissheit gibt, dass wir Mitglieder einer Gemeinschaft sind“" (G 122f.), umso sichtbarer wird im Zeichen des Kontrastes die Vereinsamung der Figuren. In der thematisierten Zuflucht in kollektive Feste kommen auf der Metaebene nicht nur zwanghafte Glückserwartungen zum Vorschein, sondern auch der gesellschaftliche Druck, von einem Angebot Gebrauch zu machen und auf diese Weise die Wünsche erfüllt zu bekommen, zumal weder das altbewährte Grenzgangszenario noch die zu besetzenden Rollen mit unangenehmen Überraschungen aufwarten.

Es sind gerade soziale Rollen, über die Thomes Protagonisten stolpern. Den narrativen Kristallisationspunkt des Stein-Kapitels bilden missglückte Lebensentwürfe der Figuren, die vor dem Geständnis, als Mann/Frau versagt zu haben, sich dadurch retten, dass sie gefühlsmäßig versteinern. „Abwesenheit von Gefühlen ist auch ein Gefühl“" (G 39), ironisiert der Mann, indes die Frau bemerkt, dass ,ab einem bestimmten Maß an Frustration das Lustempfinden [...] weder abgestorben noch am Leben ist, sondern erstarrt, wie eingefroren." (G 269) Der die Stabilität und Dauerhaftigkeit suggerierende Stein verweist hier symbolisch auf soziale Rollen als ein Fundament und ein Garant des inneren Zusammenhalts, der den Figuren in keiner Form gewahr wird.

Wenn Eva Illouz soziale Rollen in Beziehung zum Begehren bringt, hebt sie hervor, dass diejenigen, die ,nicht erst ausgehandelt werden müssen, zu spontaneren, unmittelbareren und weniger verkopften Gefühlen beitragen“. ${ }^{9}$ Vor diesem Hintergrund führt die Geschichte einer gescheiterten Beziehung der Hauptfigur fast plakativ vor, wie die signifikante Unklarheit über die Geschlechterrollen, welche das Paar immer wieder aufreibende (Wort)Kämpfe um den Beziehungskonsens ausfechten lässt, das „Verschwinden der Sehnsucht“ (G 21) bewirkt, den Niedergang des Begehrens. Auf das Spannungsverhältnis von Begehren und Männlichkeits- und Weiblichkeitsrollen gehen die Figuren in ihren Gesprächen ein: „Sei ein Mann. So hatte es Konstanze ausgedrückt“ (G 39); „Sei ein Mann, hatte sie gesagt, und nun würde er versuchen einer zu sein - bloß vielleicht ein anderer, als sie wünschte.“ (G 55) Obsessiv ruft sich Thomas die Appelle seiner Verflossenen in die Erinnerung zurück, ohne dahinterzukommen, wie das Mannsein zu konturieren wäre. Fest steht lediglich, dass seine und ihre Auffassung davon auf keinen gemeinsamen Nenner zu bringen sind. Den Anlass zum Streit bieten neben Karrierevorstellungen auch die von der gemeinsamen Zukunft. Da der Protagonist anders als die Freundin alles der Arbeit unterordnet, sind die Verwüstungen in dem Selbstbild als Mann nach dem Karriereknick - seine Habilitation wird nicht angenommen - umso größer, als der Traum von der Professur die einzige feste Koordinate darin bildet: „Er war nicht der Typ fürs Provisorium. Er führte Buch über Bücher, die er las.“ (G 73)

\footnotetext{
${ }^{9}$ Eva Illouz: Die neue Liebesordnung. Frauen, Männer und Shades of Grey. Aus dem Englischen von Michael Adrian. Berlin 2013, S. 59.
} 
Dabei beschränken sich männliche Plädoyers für dauerhafte Strukturen ausschließlich auf das Berufliche, denn im Privaten wird jeder Verbindlichkeit ausgewichen. Je öfter die Freundin vom Zusammenziehen oder vom Kinderwunsch spricht, desto fadenscheiniger werden die Vorwände. „Niemand, der heiratet, entgeht der Banalität der Ehe“ (G 168), verkündet der Mann und „stellt sich auch ehelichen Sex öde vor: Müde, missionarisch, monatlich" (G 77). Einerseits des Beziehungsstreitens überdrüssig, andererseits mit dem „Bewusstsein seiner eigenen Jämmerlichkeit“ (G 55) kehrt Thomas letztendlich sowohl der Hauptstadt als auch der Freundin den Rücken, um in der Provinz eine Singleexistenz zu führen. Es fällt dabei auf, dass Thome die Asymmetrie zwischen den Geschlechtern genau an dem Punkt festmacht, den Illouz als eine für die moderne Männlichkeit symptomatische Abneigung gegen eine langfristige emotionale Bindung beschreibt: „Emotionale Distanziertheit ließe sich als Metapher für eine männliche Autonomie verstehen, die mit der Trennung von Sex und Ehe nur noch realisierbarer wurde. “10

Wenn parallel dazu die Lebensgeschichte der weiblichen Hauptfigur erzählt wird, bestätigen deren Erlebnisse praktisch zur Gänze die männliche Befürchtung, dass alle Ehen in der Form eines Klischees enden. Vom einstigen Prinzen, der seine vom Familienleben angekratzte Männlichkeit - stereotyp - durch Motorräder und eine Jüngere aufrichtet, äußert sich die Protagonistin wie folgt: „Ein Mann, ein Wort, breite Schultern, ein Schwanz. [...] Mit anderen Worten: Ein Arschloch“" (G 95). Dabei entschied sie sich bewusst für eine traditionelle Männlichkeit, die sich selbst und ihrer sexuellen Macht sicher ist. Was sie seinerzeit an dem Mann faszinierte, war gerade dessen Selbstsicherheit, die mit sexueller Direktheit, Initiative und besitzergreifenden Gesten einherging, um Geborgenheit und Schutz zu versprechen. Beschränkt sich aber Kerstins Sehnsucht nach männlicher Dominanz auf die sexuelle Sphäre, so ist sie für den politisch ambitionierten Lokalcasanova untrennbar mit der gesellschaftlichen verbunden. Als die Frau aus dem leidenschaftlichen Traum erwacht, findet sie sich in einer Rolle vor, die sie in dieser Form niemals gewollt hat: berufliche Pläne gibt sie nach der Heirat auf, stattdessen werden mit einem obligaten Lächeln fremde Wünsche erfüllt. Da die Hausfrauen- und Mutterrolle wenig Raum für Selbstverwirklichung lässt und somit an dem Selbstgefühl zerrt, wird der Kampf um die Anerkennung und Autonomie als Frau ins Ehebett verlagert: „Sex zwischen den Eheleuten war schließlich das Normalste der Welt, aber genau genommen war es das überhaupt nicht, sondern ein kompliziertes Spiel aus Lust und Gegenlust" (G 308), stellt Kerstin im Rückblick fest. Die Logik des Begehrens steht der Logik der Autonomie insofern unversöhnlich quer, als die Leidenschaft für Kerstin nur um den Preis des Selbstverlustes zu haben ist. Dies gibt dem letzten ehelichen Sex den Anschein eines Kampfes auf Leben und Tod.

${ }^{10}$ Eva Illouz: Warum Liebe weh tut. Eine soziologische Erklärung. Aus dem Englischen von Michael Adrian. Berlin 2012, S. 143. 
Im Befund, die Sexualität sei zu einem Terrain der großen Unsicherheit gewor$\operatorname{den}^{11}$, stimmt Sigusch mit Illouz überein und argumentiert, diese sei „heute nicht mehr die große Metapher des Rausches, des Höhepunkts, der Revolution, des Fortschritts und des Glücks". ${ }^{12}$ Thome entgeht nicht, dass die mittlerweile banalisierte und kommerzialisierte Sexualität an Sprengkraft verloren hat. Mit Fragen nach dem Stellenwert der Sexualität im Selbstkonzept sowie nach Grenzen der sexuellen Freiheit werden die Hauptfiguren in Grenzgang zwei Mal direkt konfrontiert. Ihr erstes intimes Treffen findet in einer Grenzgangnacht statt, als Thomas nach der beruflichen und privaten Katastrophe in die Heimatstadt zurückkehrt und Kerstin hingegen bemerkt, dass ihr Ehemann fremdgeht. An den innigen Kuss, der ein Vorspiel hätte werden können, erinnert sich die Protagonistin wie folgt:

Weder auf seinen Lippen noch auf seiner Zungenspitze lag eine Antwort, und am stärksten empfand sie das Ausbleiben jeder Überraschung, ihre kühle Kenntnisnahme dieser Sinnlosigkeit. [...] Nichts, was er tat, war ihr unangenehm, sie glaubte bloß zum ersten Mal in ihrem Leben zu wissen, wie Frigidität sich anfühlt. Seine Lippen waren nur Lippen, seine Hände nur Hände, seine Zunge nur feucht. [...] Zum ersten Mal seit dreizehn oder vierzehn Jahren küsste sie einen anderen Mann als den eigenen und es fühlte sich ungefähr so an wie die Betrachtung der Kopie eines bekannten Gemäldes: Man schaut darauf, findet keinen Unterschied, und dennoch fehlt etwas. Zu glatt alles, die Linien zu schnell gezogen und statt Phantasie nur Fingerfertigkeit. [...] Bedeutet das, dass sich monogam bin, fragte sie sich. (G 175f.) [Hervorhebung - J.D.]

Während Thomas der Versuchung nicht widerstehen kann, mit einer attraktiven Frau den angestauten Stress abzubauen und das geschundene Ego aufzuwerten, ist für Kerstin die Umarmung, mit der sie es dem untreuen Ehemann heimzahlen möchte, nicht im Geringsten genussreich. Wie aus der zitierten Passage hervorgeht, werden nicht nur die Kälte sowie das ausbleibende Begehren nachdrücklich betont, sondern das Begehren auch explizit in Verbindung zu einer festen Beziehung gebracht. So steht der männlichen Unverbindlichkeit eine gefühlsbetonte Verstrickung der Frau gegenüber, der es sichtbar schwer fällt, die Sexualität vom emotionalen Engagement abzukoppeln. In Kerstins Vermutung, sie sei trotz ihrer Erfahrungen doch monogam, artikuliert sich die Sehnsucht, die sexuelle Freiheit mit Intimität zu verschränken, eine Sehnsucht, die Illouz zufolge auf eine komplexe Weise die Sexualität der Frau bestimme. ${ }^{13}$ Im Roman wird es umso deutlicher, wenn die Hauptfiguren einander nach Jahren zufällig in einem Swingerclub begegnen.

Nur zögernd begleitet Kerstin dorthin eine Nachbarin, deren Wunsch für sie unerklärbar bleibt, zumal weder Vernachlässigung noch Torschlusspanik auf diese zuzutreffen scheinen. „[A]lso bleibt nur die Banalität: Wir tun es, weil wir es können“ (G 265), lautet das Fazit, das eindeutig auf den spürbaren Druck abhebt, keine

\footnotetext{
${ }^{11}$ Vgl. Illouz: Die neue Liebesordnung, S. 38f.

${ }^{12}$ Sigusch: Neosexualitäten, S. 8.

${ }^{13}$ Vgl. Illouz: Die neue Liebesordnung, S. 36.
} 
Gelegenheit zum Amüsement zu verpassen. Während die Nachbarin - in einer sicheren Entfernung von der häuslichen Familienidylle - sich dem von Sigusch beschriebenen „Zwang zur Vervielfältigung und Ungezwungenheit"“14 hingibt, in der Hoffnung, mit Gruppensex das öde Wohlstandsleben aufzupeppen, versucht Kerstin, die Nacht an der Bar zu überstehen. Aus ihrer Perspektive sieht das Innere des namentlich etwas Unkonventionelles, ja Ungezwungenes suggerierenden Bohéme-Clubs folgendermaßen aus:

Trotz warmer Farben atmet der Raum etwas von der Sterilität einer Saft-Bar in einem Sonnenstudio. Die Pflanzen spenden ein eher lebloses Grün, und die kleinen Sitzgruppen entpuppen sich als Gartenmöbel aus Kunststoff. (G 291)

Die bizarre Künstlichkeit und Biederkeit des Ambiente rufen keine Assoziationen mit einer wilden sexuellen Ekstase hervor, eher schon mit sexuellem Elend. Es ist, als beschriebe Thome in der räumlichen Inszenierung Sigusch' Diagnose über verzweifelte Partnertauschpraktiken: „zeitgemäße Sumpfblüten spezifisch zerstörter Sinnlichkeit, an kleinbürgerlicher Stupidität nicht zu überbieten". ${ }^{15}$ Zugleich entspricht die Raumsterilität der mangelnden Lust der Frau: Sie „hat das Gefühl, dass der Wodka sie nicht wärmt, sondern ihr kalt im Magen liegt und ihre Gesichtszüge gefrieren lässt“ (G 297); „Kalt und eckig, wie ein langsam tauender Eisklotz, liegt ihr der Wodka im Magen. Lust hat sie keine“ (G 300).

Lust hat hingegen Thomas, der sich in einem Nebenraum anschickt, ein Tête-à-Tête mit einer seiner Internetbekanntschaften auszukosten. Nach dem Bruch mit der Freundin glaubt er, für Beziehungen unbegabt zu sein und perfektioniert die Fähigkeit, „,ein Spieler zu sein“: „, sich nicht zu verlieben, sondern neugierig zu sein. Aufmerksam, sprungbereit und unsentimental“ (G 322). Der Einladung in den Swingerclub folgt er aus zweifachem Grund. Zum einen macht die Unbekannte in den Mails einen dermaßen souveränen Eindruck, dass kein weinerliches Nachspiel zu befürchten ist, sollte der Abend kein Erfolg werden. Zum anderen sind dem Mann Anspielungen auf ein SM-Spiel insofern willkommen, als das theatralische Inszenieren des Begehrens in dieser Form hochgradig formalisiert ist und daher angstfrei erscheint. Wie Illouz ausführt, sei der Konsens bei Praktiken dieser Art im Vergleich mit sexuellen ,Normalbeziehungen' viel ernster, weil die Parameter der Erfahrung, auf die man sich einlässt, vorab sorgfältig definiert und erst dann inszeniert werden. ${ }^{16}$ So sieht der Protagonist „,den Reiz des Spiels gerade darin [...], dass es in einem eng begrenzten Feld ausgetragen wird“" (G 322).

Es ist aber nicht allein Kerstins entsetzter Schrei, als sie ihn in flagranti ertappt, der ihm den Spaß verdirbt und bewirkt, dass die Bilanz, wie bei früheren Affären

\footnotetext{
${ }^{14}$ Sigusch: Neosexualitäten, S. 86.

${ }^{15}$ Ebd., S. 16.

${ }^{16}$ Vgl. Illouz: Die neue Liebesordnung, S. 69-70.
} 
auch, recht ernüchternd ausfällt: „Ein Spiel für Verlierer, aber besser als Einsamkeit" (G 165). Es ist hauptsächlich die Scham. Ohne Scham gebe es kein Begehren, sondern allenfalls die leicht zu befriedigende sexuelle Erregung, schreibt der britische Philosoph Robert Scruton. ${ }^{17}$ Die beschämende Erfahrung im Club führt den beiden vor Augen, dass sie einander seit Langem begehren.

Im Unterschied zum spektakulär-grotesken Zwischenfall im provinziellen Swingerclub, der die kulturelle Banalisierung der Sexualität ebenso deutlich macht wie die Schwierigkeiten, das Begehren in Worte zu fassen ${ }^{18}$, wird mit einer demonstrativen Unspektakularität geschildert, wie die Figuren versuchen, der neuen „Lust der warmen, sanften Art“ (G 375) nachzugeben, dem schwer zu fassenden „Drang, zu rennen oder sein Gesicht in den blättrigen Waldboden zu drücken“ (G 233). Das Begehren führe nicht zur Liebe, aber es gebe ihr ein Motiv ${ }^{19}$, konstatiert Scruton. Es dauert lange, bis die Frau diesbezüglich bescheidene Wünsche formuliert; es dauert noch länger, bis der Mann seine Liebesbedürftigkeit zugibt und sich traut, die Maske des coolen Don Juan abzulegen, ohne den Hintergedanken, damit an Männlichkeit einzubüßen. Dem aufkeimenden Gefühl blickt er skeptisch entgegen, ohne zu wissen, ob er zu einer Nicht-Verstellung fähig wäre. Mit der offen gezeigten Zuneigung der Frau überrumpelt, wird er nach der ersten gemeinsamen Nacht das Gefühl nicht los, blindlings in eine heimtückisch aufgestellte Falle gegangen zu sein:

Und jetzt sieht er zu, wie Kerstin Werner ihm diese Schlinge um den Hals legt, als ob er vorhätte, auf seine alten Tage zum Kleinstadt-Kantianer zu mutieren: Handle so, dass die Maxime deines Willens jederzeit als Prinzip einer langweiligen Ehe gelten könne. (G 323)

„Doch alle Lust will Ewigkeit ${ }^{\text {‘20 }}$, heißt es in Nietzsches Nachtwandler-Liedern Zarathustras. Als wäre der Anspruch auf Ewigkeit der Liebe in der Literatur niemals abgestritten, klingt im Titel des letzen Romankapitels (...in Ewigkeit.) unverkennbar die Zauberformel des romantischen Narrativs an. Dennoch gleitet die Geschichte in kein billiges Happyend ab. Wenn am Ende das Ehepaar Weidmann porträtiert wird, liegen zwischen jenem Abend und der ersten, leidenschaftlichen Nacht sieben Jahre zurück. Thomas und Kerstin streiten über den nicht gekauften Paprika, als führten sie in der neuen Konfiguration seine Zweifel und ihre Erfahrungen vor: ,aus Liebe wird Routine, aus Routine Langeweile, aus Langeweile Streit" (G 215). Die in den Ehealltag eingeblendeten Bilder von Kerstins blindem Rausch, als sie den ersten Mann kennenlernte, sowie die Szenen, als Thomas der Ex-Freundin offen ins Ge-

\footnotetext{
${ }^{17}$ Vgl. Roger Scruton: Pożądanie. Filozofia moralna życia erotycznego. Przekład: Tomasz Kuniński. Kraków 2009, S. 251 [Sexual desire. A moral philosophy of the erotic].

${ }^{18}$ Thomas wundert sich, „,dass Liebe so ein autistisches Gefühl sein kann. Beinahe unaussprechlich.“ (G 430)

${ }^{19}$ Vgl. Scruton: Pożądanie, S. 281.

${ }^{20}$ Friedrich Nietzsche: Also sprach Zarathustra. Kritische Studienausgabe Bd. 4. Hrsg. von Giorgio Colli und Mazzino Montinari. München 1999, S. 401-402.
} 
sicht sagt: „,nach Abzug der Hormone sei Liebe schließlich auch nur eine besonders virtuose Form von Geduld“ (G 368), nehmen allen Liebeserklärungen, Hoffnungen und den versöhnenden Gesten ihren Glücksimperativ ab. Die in den ironischen, desillusionierten Bemerkungen greifbare Abgeklärtheit, die auf die gegenwärtige Entzauberung der Liebe hindeutet, lässt im Zusammenhang mit dem bevorstehenden Grenzgang-Fest umso deutlicher an den ewigen Teufelskreis der Bemühungen um einen Beziehungskonsens denken, an die Frage, ,wie man ein freiwillig gegebenes Begehren in einer ansonsten völlig konsensuellen Beziehung auf Dauer sicherstellen kann. “21

\section{2.}

Die Frage bildet den roten Faden in Thomes nächsten Romanen. In Fliehkräfte geht es um das Scheitern einer Ehe, das aus der Perspektive des Hauptprotagonisten Hartmut Hainbach erzählt wird. Dem Endfünfziger gelang etwas, worum sich der Protagonist in Grenzgang umsonst bemühte - sich von provinziellem Herkunftsmilieu zu emanzipieren und ordentlicher Professor zu werden. Anders als Thomas verschanzt sich Hartmut hinter der Idylle familiären Glücks, die zusammenbricht, als die Tochter das Haus verlässt und die Ehefrau Maria nach Berlin geht, um dort beruflich Fuß zu fassen. Auf eine Wochenendbeziehung reduziert, steuert die Ehe unwiderruflich ihrem Ende zu, zumal Maria nach Jahren der hausfraulichen Öde die letzte Chance auf Selbstverwirklichung nicht verpassen will. Als Hartmut ein Stellenangebot aus einem Berliner Fachverlag bekommt, sieht er sich vor die Entscheidung gestellt, der Ehe zuliebe einen Neuanfang zu wagen. Einerseits lockt dieser mit dem Versprechen der Zweisamkeit an, andererseits birgt er Gefahren in sich. Das Aufgeben der Professur bedeutet nicht nur, eine Identitätsstütze zu verlieren, sondern auch finanzielle Einbußen hinnehmen zu müssen. Mithin wäre der Preis für die emotionale Sicherheit mit dem Autonomieverlust verbunden, und dies ohne zu wissen, ob die Aufopferung von der Frau überhaupt angenommen wird.

In dem drei Jahre später veröffentlichten Roman Gegenspiel wird die Geschichte noch einmal aufgegriffen, diesmal aus der Sicht von Maria. Der Autor macht das Prinzip des kontrapunktischen Erzählens produktiv, mit dem zuvor Hartmuts Werdegang auf den akademischen Olymp und zum stolzen Ehemann inszeniert wurde, um zu schildern, wie die Verwandlung einer künstlerisch ambitionierten Frau zu einer deprimierten Mutter und Hausfrau verläuft, welche vergrämt dem Mann beim Karrieremachen zuschaut. Durch die Verschränkung zweier Perspektiven - der tristen Gegenwart mit vormaligen Hoffnungen - werden in dem Romandoppel die nebeneinander stehenden Gegensätze aufeinander bezogen: Wo auf der einen Seite

\footnotetext{
${ }^{21}$ Illouz: Die neue Liebesordnung, S. 69.
} 
männliche/weibliche Träume von der beruflichen und erotischen Erfüllung stark gemacht werden, kommen auf der anderen Seite Enttäuschungen und Erosionsprozesse in der Ehe zum Vorschein, die nach Marias Umzug nach Berlin das ganze Ausma $\beta$ des Unheils hinter dem idyllischen Schein der bürgerlichen Wohlstandsexistenz ans Licht befördern - von den Differenzen in der Lebensplanung über die mangelnde Kommunikation und fehlende Kompromissbereitschaft bis hin zur beiderseitigen Untreue.

Obwohl man die Romane separat lesen kann, gewinnen die Chroniken einer scheiternden Beziehung ihre Kraft gerade durch die Spiegelung. Denn so unterschiedlich die Ehepartner auch sind, geht es in ihren Paralleluniversen um dasselbe die Selbstfindung und Aushandlung der eigenen Rolle, den fortschreitenden Zerfall verbindlicher Strukturen sowie um Liebe, die mit dem Drang nach Autonomie schwer vereinbar ist. Trotz thematischer und formaler Ähnlichkeiten mit Grenzgang werden die Akzente anders gelegt. Die Differenzen betreffen zum einen die Konstruktion der Figuren, zum anderen die , atmosphärische' Gestaltung des Begehrensraumes.

Ähnlich wie die Figuren in Grenzgang sind auch Hartmut und Maria ein Produkt ihrer eigenen Vorstellungen, die an der Wirklichkeit zerbrechen. Die kleinen Verletzungen in der Ehe, die sich über die Jahre negativ summieren und mit einem Desaster enden, haben ihren Ursprung in den sozialen Rollen. Bis zu dem großen Krach wirkt das Ehepaar gleichermaßen stereotyp wie unzeitgemäß: sie - eine Hausfrau, die aus Langeweile hin und wieder Sprachkurse leitet; er - ein Alleinverdiener, der arbeitet, um ihre Langeweile zu finanzieren. Das Bild, das Hartmut als Ehemann abgibt, entspricht der archetypischen, Schutz bietenden Männlichkeit, die laut Eva Illouz zwar grundsätzlich mit der Ungleichheit in der Mann-Frau-Relation einhergehe, der Relation aber gerade durch die Asymmetrie zwischen beschützendem Mann und abhängiger Frau durchaus angenehme Aspekte verschaffe: Die von der Machtin ein Beschützerverhältnis umgewandelte Ungleichheit erzeuge einen starken emotionalen ,Klebstoff ${ }^{`}$, darüber hinaus gebe es Klarheit bezüglich der Rollen, die keiner Aushandlung bedürften. ${ }^{22}$ Indem Hartmut bewährten Szenarien folgt, fühlt er sich in seiner Rolle gut aufgehoben, ohne zu bemerken, dass er sein Wohlgefühl auf die Frau projiziert. Als sie aus der fatalen Abhängigkeit ausbricht, wirft sie dem Mann an den Kopf: „Unser Leben ist die Parodie unserer Träume“. ${ }^{23}$

Im Gegensatz zu Grenzgang zeigt Thome diesmal die Verwirrung der Figuren in einer Rückkopplung auf Muster, mit denen sie in der Sozialisation konfrontiert werden. Sowohl Hartmuts als auch Marias Erwartungen konstituieren sich in einer Spannung zwischen traditionellen Vorbildern und jenen Mustern, die im Zuge der

\footnotetext{
${ }^{22}$ Vgl. Illouz: Die neue Liebesordnung, S. 58-60.

${ }^{23}$ Stephan Thome: Fliehkräfte. Roman. Berlin 2012, S. 455. In der Folge als F mit einfacher Seitenzahl zitiert.
} 
Studentenrevolte an Bedeutung gewinnen. Die Figuren stammen aus anderen Kulturkreisen und sind keine Gleichaltrigen (Maria ist um zehn Jahre jünger), dennoch werden sie in derselben Weise von der Gier nach Bildung und freiem Leben angetrieben, um beengte Verhältnisse samt Rollenvorgaben hinter sich zu lassen: er - das biedere Haus in der deutschen Provinz mit dem kalten Vater, der dem proletarischen Männlichkeitsideal der Nachkriegszeit huldigt und den Sohn am liebsten in seine Fußstapfen treten sieht, sie - ihre erzkatholische Kleinfamilie in Portugal und einen kleinkarierten Freund, der sehnlichst auf baldige Heirat hofft.

Die Konfrontation mit neuen Modellen erfolgt während des Studiums in Berlin, freilich unter einem anderen Vorzeichen. Anders als Hartmut stürzt sich Maria frenetisch auf neue Möglichkeiten und wirkt dabei beinahe wie eine Parodie weiblicher Selbstverwirklichung - mit dem Leben in einer Wohngemeinschaft, wo die Familie als „die Keimzelle des Faschismus“ ${ }^{\text {‘24 }}$ ausdiskutiert wird, der Lektüre von Simone de Beauvoir sowie mit einem exzentrischen, theaterbesessenen Freund Falk startet die junge Frau ihren Protest gegen etablierte Konventionen und die kleinbürgerliche Moral. Diesmal offen, denn sexuell emanzipiert sich die Musterschülerin einer katholischen Bildungsanstalt noch in Portugal, indem sie sich auf eine Affäre mit einem älteren Mann aus besseren Kreisen einlässt. Wiewohl diese mit einer ungewollten Schwangerschaft und einer illegalen Abtreibung endet, lernt Maria von dem erfahrenen Liebhaber, stolz auf ihren Körper zu sein sowie die Sexualität als lustvoll und ohne Schuldgefühle zu erleben.

Thome schildert beide Protagonisten in Widersprüche verfangen, die - folgt man der These von Illouz - aus der Verfassung des modernen Liebeslebens resultieren: der Autonomiegewinn der Sexualität habe das Feld der emotionalen Interaktionen hochgradig verunsichert und mit zwiespältigen Gefühlen bezüglich Regeln zum Aushandeln von Bindungen, Liebe und Begehren erfüllt. ${ }^{25}$ In der Souveränität, mit der Maria die sexuelle Initiative in der Beziehung zu Falk ergreift - „Ich will öfter mit dir schlafen. Wir tun es zu selten" (GS 150) - erkennt man das Kind seiner Zeit. Der Freund erscheint ihr in seiner intellektuellen Pose sowie in seinem Nonkonformismus als das Ideal eines Mannes, doch reicht Maria die Mixtur aus Gesprächen, erotischer Spannung und dem Bewusstsein, gesellschaftliche Tabus zu brechen, auf die Dauer nicht aus:

Ihr Verlangen wuchs mit der Intensität ihrer Zuneigung, schwankte dem Verlauf ihres Zyklus entsprechend und nahm den verspäteten Frühling vorweg. Seines hing von der Arbeit ab, und manchmal glaubte er sie bestrafen zu müssen, weil sie ihn zu einer ideologisch verdächtigen Lebensform überredet hatte, der Kohabitation als Paar. (GS 133)

\footnotetext{
${ }^{24}$ Stephan Thome: Gegenspiel. Roman. Berlin 2015, S. 45. In der Folge als GS mit einfacher Seitenzahl zitiert.

${ }^{25}$ Illouz: Die neue Liebesordnung, S. 70.
} 
Der überzeugte Revolutionär lehnt jeden Anschein des bürgerlichen Lebens als zwanghaft $a b$ - „Ein richtiges Ehebett, hatte er gehöhnt, nachdem sie das Geld für den Lattenrost verdient, ihn bestellt und die Lieferung organisiert hatte“ (G 143), was die Verbindlichkeit ausschließt: „Ich hab dir von Anfang an gesagt, dass Beziehungsgedöns nichts für mich ist." (GS 146) Mit ihrer wachsenden Zuneigung sehnt sich Maria vergeblich nach Sicherheit und Geborgenheit: „Ich wünschte, $d u$ würdest auf mich achtgeben." (GS 148, kursiv im Original) Die verweigerte intime Zweisamkeit spiegelt sich in der frostigen Atmosphäre der gemeinsamen Wohnung wider: „Kälte und Stille empfingen sie in der Wohnung. Das Feuer im Ofen war ausgegangen, sie würde Kohlen schleppen und drei Stunden warten müssen, bevor sie ohne Jacke am Schreibtisch sitzen konnte.“ (GS 173)

Vor der Kälte flieht Maria in die Arme von Hartmut, der nicht nur mit den Einladungen „,zu einer warmen Mahlzeit“ (GS 167) die herbeigewünschte „,warme Intimität“ (GS 55) zu versprechen scheint. Glücklicher wird sie nach der Heirat jedoch nicht, denn die Liebe allein reicht nicht aus, um alle Fallen in einer Beziehung aus dem Weg zu räumen. „Sagen wir, es war nie mein Plan, in der weiblichen Dreieinigkeit aufzugehen. [...] Ehefrau, Hausfrau und Mutter, das wollte ich tatsächlich nicht“" (GS 405), rechtfertigt sie der erwachsenen Tochter gegenüber ihre Depression und die Entscheidung, nach zwanzig Ehejahren allein nach Berlin zu gehen. Weder will sie sich von Hartmut trennen, noch ihn bestrafen, vielmehr glaubt sie, das in der widerwillig bewältigten Alltagsroutine abhanden gekommene Begehren aufrechterhalten zu können: „Wir werden uns seltener sehen, aber dann mit Zeit füreinander und Lust aufeinander. Es war schön, als du in Dortmund gewohnt hast und ich in Berlin. Oder nicht?" (GS 24)

Hartmuts Skepsis der räumlichen Distanz gegenüber rührt daher, dass er eine solche Option bereits kennt. Aus seiner ersten Liebe wird eine lebenslange Freundschaft, denn zu mehr reicht es wegen der Entfernung nicht, als die in den USA kennengelernte Französin ihr Stipendium verlängert und auf sich warten lässt. Die Wartezeit überbrückt Hartmut mit unverbindlichen Affären, zumal Enthaltsamkeit wenig mit dem Zeitgeist in den Kreisen korrespondiert, in denen er in den 1980er Jahren in Berlin verkehrt. Dass die sexuelle Entfesselung jedoch mit zum Teil erheblichen Fremd- und Selbstzwängen einhergeht, wird in der Einstellung einer seiner Freundinnen manifest, von der es heißt: „Sie war katholisch, promovierte über Befreiungstheologie und gab sich so freizügig, weil sie glaubte, dass es von ihr erwartet wurde.“ (G 176) Auch für Hartmut mutiert das vorherrschende Verständnis der Lustoptimierung immer mehr zu einer Zwangsbeglückung: „Er ist das Dauerbumsen leid, diese horizontalen Überstunden, die schon lange kein Abenteuer mehr sind.“ (F 176)

„Im Grunde tickst du wie die frommen Bauern in dem Kaff, aus dem du kommst. Für dich bin ich bloß ein notgeiles Miststück“ (F 176), macht ihm eine verheiratete Geliebte zum Vorwurf, als er aus der Ménage-à-trois aussteigt. Dass 
Frustration der Lohn einer mühsamen Befreiung von gesellschaftlichen Tabus ist, leuchtet Hartmut ein, als der dem experimentierfreudigen Mann seiner Geliebten von Angesicht zu Angesicht gegenübersteht. Mit der Parole „Ich assoziiere Bärte übrigens mit Männlichkeit“ (F 174) zeigt sich der einvernehmlich Betrogene unmissverständlich als ein Kulturrevolutionär von einst, wenn auch in der Version „Macho als mürrischer Veteran“. ${ }^{26}$ Vom einstigen revolutionären Männlichkeitsideal bleibt die Fassade erhalten, hinter habituellen Ausdrucksformen wie der rebellischen Antifrisur - dem Sinnbild unverbrauchter Natürlichkeit und sexueller Allmacht, welche systemkonforme Kleinbürger herausfordern sollte - verbergen sich jedoch keine Gesten der Entschlossenheit mehr, sondern Verzweiflung und Resignation. Der in eine Küchenschürze gewickelte Mann sieht nicht aus, als würde er „gleich aufspringen und die Revolution ausrufen“: „Statt den Sturm auf die Bastille anzuführen, fragt Klaus nach dem Zustand der Klöße“ (F 171). Ohne entscheiden zu können, „ob er $\mathrm{Zu}$ - oder Abneigung empfindet, Achtung oder Mitleid“ (F 171), nimmt Hartmut wahr, die Befreiung aus gesellschaftlichen Normen verschaffe dem Gegenüber nur noch Depression.

Die Zwischenstationen zeichnet der Autor mit wenigen Strichen, durch die zeitliche Verortung einzelner Erinnerungssequenzen wird aber außer den Konturen von der emotionalen Grammatik gegenwärtiger Männlichkeit und Weiblichkeit auch die Vielfalt der Beziehungsformen umrissen. Die Figuren irren von einem Konzept zum nächsten, um zu erfahren, dass keines das richtige sei, dass - mit Sigusch gesprochen - alle derzeit kursierenden Intimbeziehungen „dieselbe Maske des falschen Glücks [tragen], wie ehedem die eheliche Liebe ${ }^{\text {‘27 }}$. Mal versuchen die Protagonisten, sich von einem Model abzugrenzen, mal Anschluss daran zu finden, doch unabhängig davon, wie stark der jeweilige Mimikrywunsch ist, werden sie wie bei der Fliehkraft stets nach außen geschleudert, in Positionen, die Widersprüchliches in sich vereinen. Als ein „Achtundsechziger“ (F 353) hängt Hartmut an bürgerlichen Normen; als ein Befürworter der Frauenemanzipation blüht er in alten Rollenzuweisungen auf; als Romantiker, der wider den Zeitgeist in der Verbindlichkeit eine Tugend erkennt, betrügt er gelegentlich die Ehefrau mit der französischen Ex-Freundin. Entsprechend kann die freiheitsbesessene Maria emotional weder über die Abtreibung noch über ihre (einmalige) Untreue hinwegkommen: „Nie hat sie sich den Seitensprung verziehen - auch nicht, als sie sicher war, genug für ihn gebüßt zu haben" (GS 455).

Damit ist nicht gemeint, dass alle Perspektiven in ihrer Differenz den Protagonisten gleich wertvoll sind, was nicht zuletzt in der atmosphärischen Gestaltung der

${ }^{26}$ Aribert Reimann: Zwischen Machismo und Coolness. Männlichkeit und Emotion in der westdeutschen ,Kulturrevolution ' der 1960er und 1970er Jahre. In: Manuel Borutta, Nina Verheyen (Hrsg.): Die Präsenz der Gefühle: Männlichkeit und Emotion in der Moderne. Bielefeld 2010, S. 229-253, hier S. 244.

${ }^{27}$ Sigusch: Neosexualitäten, S. 40. 
Begehrensräume zum Ausdruck kommt. Thome funktionalisiert die Kälte/WärmeMetaphorik auf zweifache Weise - zum einen im Zusammenhang von Begehren und Geschlecht. Die auf der Figurenebene arrangierte Konfrontation von Mann und Frau ruft unweigerlich Assoziationen von Rationalität/Emotionalität, Kälte/Wärme hervor. In Fliehkräfte und Gegenspiel sticht es besonders ins Auge, weil Hartmut die Rationalität geradezu zu verkörpern scheint - als Philosophieprofessor wird er zum Inbegriff eines Denkers, der den Gelehrtenhabitus selbst in seinen vier Wänden wahrt. In Marias Augen ist er ,ein ruhiger, kontrollierter Mensch, der seine wunden Punkte zu verbergen weiß" (GS 14). Sie wiederum scheint diejenige zu sein, die sich von Affekten leiten lässt, und sei es bis zum Äußersten: „Zum ersten Mal im Leben hat sie ihren Mann geschlagen, aber der Schock lässt kein Nachdenken darüber zu.“ (GS 18) Die exponierte Gegenüberstellung von der personifizierten Kälte und Wärme, durch die geografische Markierung kulturell geprägter Schemata zusätzlich verstärkt (kühler, reservierter, Nordeuropäer/heißblutige Südländerin), mutet an, als würde Thome allerhand Stereotype bemühen, um geschlechtsspezifische Temperaturen des Begehrens auf den Punkt zu bringen.

Dem ist keinesfalls so. Gerade durch die Zuspitzung wird eine solche Konstellation von Temperatur und Geschlecht als klischeehaft desavouiert. Dass die Temperaturwerte des Begehrens individuell gemessen werden müssen, zeigt sich daran, dass es nicht möglich ist, die Figuren auf eine Reihe eindeutiger Merkmale festzulegen. So sehr Hartmuts Habitus sachlicher Kälte mit klassischen Männlichkeitsbildern harmoniert, so wenig passt hierzu seine, wie Illouz ausführt, traditionell feminin konnotierte, brennende „Sehnsucht nach einer emotionalen Symbiose“. ${ }^{28}$ Anders als die Frau vermutet, verbirgt sich hinter der Kälte-Maske eine innere Leidenschaft, für die der Mann keinen passenden Ausdruck findet, die ihn aber nach Marias Umzug stark leiden lässt - der physischen Distanz kann er nichts abgewinnen, weil sie ihm der emotionalen gleichkommt:

Ihn haben die vergangenen zwei Jahre gelehrt, dass Liebe ein schwaches Argument sein kann. Schwächer als einsame Nächte, die Frustration über ihr abgeschaltetes Handy oder das merkwürdige Gefühl beim Betreten von Marias Wohnung. (F 40)

Umgekehrt bemüht sich die temperamentvolle Maria um die kühle Sachlichkeit - sie kämpft um ihre Autonomie, davon überzeugt, die Ehe profitiere von ihrer Selbstständigkeit, und sei diese auch andernorts unter Beweis gestellt. Bei dem Kälteschild, hinter dem sie sich versteckt, geht es allerdings weniger um die Abwehr der Gefühle, denn um die Abwehr der Gefahr, dem Mann ausgeliefert zu sein.

Betrachtet man die Kälte/Wärme-Metaphorik der Romane - zum zweiten - im Kontext der Beziehungserfahrungen der Figuren, merkt man an den meteorologischen und topographisch-räumlichen Koordinaten, dass beide Figuren wegen frühe-

\footnotetext{
${ }^{28}$ Illouz: Die neue Liebesordnung, S. 45.
} 
rer Enttäuschungen gleichermaßen an der Verbindlichkeit des Projekts ihrer Liebesehe hängen. Im Unterschied etwa zu einem „klirrend kalten Wintertag“ (GS 56), an dem Maria Abschied von dem Ex-Freund nimmt, oder zur Kälte des Kellerraumes, in dem sie in der postnatalen Depression einen zufälligen Sex hat, strahlt „ein verheißungsvoll sonniger Himmel“ (F 29) selbst dann, wenn die Eheleute bei einem der immer selteneren Wochenendetreffen in Berlin gemeinsam frühstücken. So verheiBungsvoll die inszenierte sommerliche Hitze in Portugal, wo sie einst zum Paar wurden, und wo sie sich zu einem Gespräch über die gemeinsame Zukunft treffen sollen, auch anmutet, lässt der Autor die Paradoxien des Begehrens nicht aus den Augen: Indem Hartmut sehnsüchtig auf Maria wartet, holt er sich einen schweren Sonnenbrand. Anstatt für Klarheit zu sorgen, unterbricht er dann schlagartig das Gespräch, geht schwimmen und lässt die bestürzte Frau zurück mit den Worten: „Vielleicht solltest du dich auch abkühlen.“ (GS 452)

\section{3.}

,$\ldots$ das will ich festhalten. Die Veränderung. Die sich übrigens nicht festhalten lässt, aber es lohnt den Versuch.“ (F 143) Der Satz, mit dem der Hauptprotagonist in Fliehkräfte erklärt, warum er ein Tagebuch führe, klingt wie das poetologische Credo von Stephan Thome. Als ,unerbittlicher Berichterstatter vom Schlachtfeld der Liebe ${ }^{\text {“29 }}$ richtet der Autor die erzählerische Aufmerksamkeit auf die Vielfalt moderner Beziehungsformen und inszeniert dabei den von Volkmar Sigusch konstatierten „eher lautlosen Wandel“ ${ }^{\text {*30 }}$ - den kulturellen Abgesang auf die Sexualität als die Glücksmetapher schlechthin und das Aufgehen der triebhaften Wollust in eine Wohllust exklusiver Intimität.

Behauptet der Sexualwissenschaftler, über allem throne die als eine einzigartige Kostbarkeit fetischisierte Liebe ${ }^{31}$, so entgeht dem Schriftsteller nicht, dass die inzwischen entzauberte, rationalisierte Liebe mächtiger ,weh tut“ (Eva Illouz) als je zuvor. Mag die Wärme der Liebesbeziehungen den Figuren als ein spezifisches Antidot gegen die gesellschaftliche Kälte vorkommen, so zeigt sich dieses als dürftig: mal frieren sie in gebauten Luftschlössern, mal bekommen sie in der brütenden Hitze einen Sonnenstich. In diesem Sinne weist der Satz, mit dem die Protagonistin in Grenzgang nach dem Paprikastreit ihr Wohlbefinden kommentiert, metaphorisch auf Temperaturwerte des - prekären - Begehrens hin: „Es gibt Tage, da erinnern die Schwankungen ihrer gefühlten Körpertemperatur an ein Börsenbarometer" (G 409).

${ }^{29}$ Thomas Andre: Beziehungskrisen: Erst kommt die Ehe, dann das Elend [online], <http://www. spiegel.de/kultur/literatur/stephan-thomes-roman-gegenspiel-bei-suhrkamp-a-1015675.html> [29.11.2015].

${ }^{30}$ Sigusch: Neosexualitäten, S. 28.

${ }^{31}$ Ebd., S. 8. 


\section{Literatur}

Andre, Thomas: Beziehungskrisen: Erst kommt die Ehe, dann das Elend [online], <http://www.spiegel. de/kultur/literatur/stephan-thomes-roman-gegenspiel-bei-suhrkamp-a-1015675.html>

Angerer, Marie-Luise: Vom Begehren nach dem Affekt. Zürich/Berlin 2007.

Illouz, Eva: Warum Liebe weh tut. Eine soziologische Erklärung. Aus dem Englischen von Michael Adrian. Berlin 2012.

Illouz, Eva: Die neue Liebesordnung. Frauen, Männer und „Shades of Grey“. Aus dem Englischen von Michael Adrian. Berlin 2013.

Nietzsche, Friedrich: Also sprach Zarathustra. Kritische Studienausgabe Bd. 4. Hrsg. von Giorgio Colli und Mazzino Montinari. München 1999.

Reimann, Aribert: Zwischen Machismo und Coolness. Männlichkeit und Emotion in der westdeutschen 'Kulturrevolution' der 1960er und 1970er Jahre. In: Manuel Borutta, Nina Verheyen (Hrsg.): Die Präsenz der Gefühle: Männlichkeit und Emotion in der Moderne. Bielefeld 2010, S. 229-253.

Scruton, Roger: Pożądanie. Filozofia moralna życia erotycznego. Przekład: Tomasz Kuniński. Kraków 2009.

Sigusch, Volkmar: Neosexualitäten. Über den kulturellen Wandel von Liebe und Perversion. Frankfurt a. M./New York 2005.

Struck, Lothar: Provinzkritiker. Aufschlußreiche Bemerkungen zu einer Pseudokritik über Stephan Thomes Buch Grenzgang, die den Zustand der bundesdeutschen Literaturkritik beleuchten [online], <http://www.glanzundelend.de/Artikel/artikelalt/provinzkritiker.htm>

Thome, Stephan: Grenzgang. Roman. Frankfurt a. M. 2009.

Thome, Stephan: Fliehkräfte. Roman. Berlin 2012.

Thome, Stephan: Gegenspiel. Roman. Berlin 2015. 